\title{
PVA Derivative Membrane Selective to Oxygen from Nitrogen Gases
}

\author{
Yoshio Taniguchi and Shinkichi Horigome \\ Central Research Laboratory, Hitachi Ltd., \\ Kokubunji, Tokyo 185, Japan. \\ (Received February 25, 1975) \\ KEY WORDS Membrane / Permeation / Selectivity / Poly(vinyl
alcohol) / Oxygen /
}

Oxygen and nitrogen gases have been grouped together because their physical properties are very similar. Up to the present day all organic polymer membranes have been found to show a 2-5 times greater permeability to oxygen than to nitrogen. ${ }^{1}$ The polymer which shows the greatest permeability towards oxygen and nitrogen is poly(dimethyl siloxane) membrane. But, its permeability ratio of oxygen to nitrogen is about $2 .^{2}$

On the other hand, a facilitated transport process is currently being applied to gas separations. This is a process in which the transport of solute across a liquid membrane is augmented as a result of a reversible reaction with a carrier which goes to and fro between opposite faces of the membrane. Several biological substances are being used in this process as carriers. There has already been a study concerning a millipore membrane charged with a hemoglobin solution. ${ }^{3}$

In this paper, we suggest a new oxygen gas permselective membrane using a new organic polymer.

In order to raise the selectivity of oxygen permeation; some organic groups which react reversibly with oxygen are combined with a polymer molecule whose membrane has very low

Table I. Gas permeability coefficients in PVA membrane at $20^{\circ} \mathrm{C}^{5}$

\begin{tabular}{|c|c|c|}
\hline Gas & $\begin{array}{l}\text { Perm. coef., } \\
\frac{\mathrm{cc}(\mathrm{STP}) \mathrm{cm}}{\mathrm{cm}^{2} \mathrm{sec} \mathrm{cmHg}}\end{array}$ & $\begin{array}{l}\text { Ratio of perm., } \\
\qquad P_{\mathrm{O}_{2}} / P_{\mathrm{X}}\end{array}$ \\
\hline Oxygen & $5.2 \times 10^{14}$ & - \\
\hline Nitrogen & $4.5 \times 10^{14}$ & 1.2 \\
\hline Helium & $33 \times 10^{14}$ & 0.16 \\
\hline
\end{tabular}

gas permeability. We chose $N, N$-dimethyl aniline (DMA) as an organic group which reacts reversibly with oxygen. The molecular complex is formed by the weak interaction of DMA with oxygen molecules. ${ }^{4}$ This complex dissociates in a phase under a low oxygen partial pressure. Poly(vinyl alcohol)(PVA) was chosen as a polymer molecule whose membrane has very low gas permeability. The gas permeability coefficients of PVA $^{5}$ are shown in Table I. In order to combine PVA with DMA groups, we adopted the acetalization of PVA with p-dimethylaminobenzaldehyde, which is the aldehyde derivative of DMA. This reaction is shown in Figure 1.

PVA derivative was synthesized in the presence of sulfuric acid catalyst. The PVA used here was type 224 produced by Kuraray Co., Japan. The PVA derivative was purified by a solutionprecipitation cycle. It was confirmed by the measurement of ultraviolet-visible and infrared absorption spectra that this product contained benzene rings but no aldehyde groups. The content of acetal of this product was estimated to be about $70 \%$ from the contents of carbon and nitrogen, on the basis of quantitative elemental analysis.

The hexamethylphosphorictriamide solution saturated with this PVA derivative was cast on a polyethylene sheet. A rubber-like membrane was obtained after drying in vacuo at $55^{\circ} \mathrm{C}$ for 2 days. This membrane was about $20 \mu \mathrm{m}$ thick.

The instrument for gas permeation measurement was driven by the pressure difference between atmospheric and diminished pressures; gas permeability coefficients were calculated from 


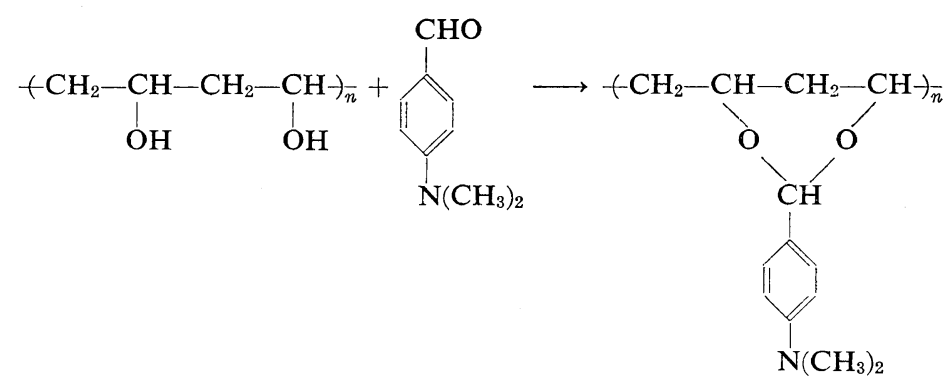

Figure 1. Reaction of PVA derivative formation.

Table II. Gas permeability coefficients in PVA derivative membrane at room temperature

\begin{tabular}{lcc}
\hline \multirow{2}{*}{ Gas } & $\begin{array}{c}\text { Perm. coef., } \\
\mathrm{cc}(\mathrm{STP}) \mathrm{cm}\end{array}$ & $\begin{array}{c}\text { Ratio of perm., } \\
P_{\mathrm{O}_{2}} / P_{\mathrm{X}}\end{array}$ \\
\cline { 2 - 3 } & $\mathrm{cm} \mathrm{sec} \mathrm{cmHg}$ & - \\
\hline Oxygen & $2.1_{3} \times 10^{10}$ & 5.8 \\
Nitrogen & $0.36_{8} \times 10^{10}$ & 0.21 \\
Helium & $10.3 \times 10^{10}$ & $0.3 \times$ \\
\hline
\end{tabular}

the pressure change in the low pressure region. ${ }^{6}$ Pure gases were used as permeative gases.

The results of gas permeation of the PVA derivative membrane at room temperature are shown in Table II. According to these results the membrane permeation ratio of oxygen to nitrogen is 5.8 , and the gas permeability coefficients of the PVA derivative membrane are increased about four orders of magnitude over those of the PVA membrane.

The permeability to helium is about five times as large as that to oxygen. This result suggests that the mechanism of gas transport through this membrane would be different from that of polydimethyl siloxane membrane, whose the permeability ratio of oxygen to helium is about 0.6. We think this PVA derivative membrane would be particularly interesting from the viewpoint of the mechanism of gas permeation through polymer membranes. More details of the PVA derivative membrane and the mechanism of permselectivity will be published soon.

\section{REFERENCES}

1. T. Nakagawa, Japan Plastics, 24 (12), 9 (1973).

2. W. L. Robb, General Electric R. \& D. Center Report, No. 65-C-031, (1965).

3. D. F. Scholander, et al., Science, 131, 585 (1960).

4. M. Hori, Bull. Chem. Soc. Japan, 43, 3765 (1970).

5. T. Nakagawa, Proceeding of 11 th Symposium on Polymer and Water, Japan, 1973, p 32.

6. Y. Taniguchi and S. Horigome, to be published. 